\title{
La transposición didáctica de la investigación en humedales
}

\author{
Beatriz García Fernández \\ Universidad de Castilla-La Mancha (España) \\ Beatriz.Garcia@uclm.es \\ Gema SÁNChEz EMETERIO \\ Universidad de Castilla-La Mancha (España) \\ Gema.Sanchez@uclm.es \\ David SÁNCHEZ RAMOS \\ Universidad de Castilla-La Mancha (España) \\ David.SanchezRamos@uclm.es
}

Recibido: 31/07/2012

Aceptado: 22/10/2012

\section{Resumen}

Se plantea una propuesta de transposición didáctica al aula con las investigaciones realizadas sobre humedales y el papel de los medios de comunicación como elementos clave. La metodología empleada se basa en la creación de una comunidad de aprendizaje donde se fomente la comunicación y se produzca un aprendizaje significativo en entornos naturales. Para ello se utilizan los medios de comunicación frecuentados por los alumnos como fuente de información motivadora, especialmente las redes sociales. La importancia de utilizar los conocimientos científicos en la docencia resulta especialmente interesante en la redefinición de la figura del maestro docente investigador de las Facultades de Educación. El objetivo final de la propuesta es hacer más eficiente el trinomio investigación-enseñanza-aprendizaje, educando a personas competentes que contribuyan a la mejora del entorno en que se desenvuelven.

Palabras clave: Transposición didáctica, humedales, comunicación, educación, fuentes de información.

\section{Didactic transposition of wetlands research}

\begin{abstract}
This article is a proposal of didactic transposition into the classroom of the investigations conducted on wetlands and the role of the media as key elements. The methodology used is based on the creation of a learning community, where encouraging communication and meaningful learning occurs in natural environments. Frequented by students as a motivating information source, media like social networks are especially used.

The importance of using scientific knowledge in teaching is especially interesting in the redefinition of the figure of new researcher teacher in faculties of education. The final objective of the proposal is to make more efficient the trinomial researching-teaching-learning, educating competent people that contribute to the improvement of the environment in which they develop.
\end{abstract}

Key words: Didactic transposition, wetlands, communication, education, information sources.

\section{Referencia normalizada}

GARCÍA FERNÁNDEZ, Beatriz; SÁNCHEZ EMETERIO, Gema y SÁNCHEZ RAMOS, David (2012): "La transposición didáctica de la investigación en humedales". Estudios sobre el mensaje periodístico. Vol. 18, núm. especial octubre, págs.: 347-356. Madrid, Servicio de Publicaciones de la Universidad Complutense.

Sumario: 1. Introducción. 2. Metodología y desarrollo; 2.1. Los humedales de la Reserva de la Biosfera de La Mancha Húmeda; 2.2. Los humedales en la formación docente: transferencia de conocimiento; 2.3. Las redes de comunicación en la transferencia de conocimientos. 3. Conclusiones. 4. Referencias bibliográficas. 


\section{Introducción}

El presente artículo constituye una reflexión, con aportaciones teóricas de aplicación práctica, sobre la transposición didáctica de aquellos resultados obtenidos en las investigaciones en el ámbito de los humedales a las Facultades de Educación. En este proceso de transposición didáctica (término acuñado por Michel Verret en 1975), la importancia de los medios de comunicación es clave frente al objetivo de hacer más eficiente el trinomio investigación-enseñanza-aprendizaje, siendo uno de los objetivos de estas reflexiones que los futuros docentes sean capaces de trasladar, a su vez, dichos conocimientos al aula de Educación Primaria.

El conocimiento fruto de las investigaciones científicas, tiene que sufrir un proceso de reelaboración antes de ser llevado a las Facultades de Educación, para poder ser aprovechado en la docencia. Los medios en que estos conocimientos se transmiten no deben limitarse a aquellos proporcionadas por el docente, sino que deberán ser motivadores, fomentando el mejor aprendizaje de los conocimientos, y la disponibilidad para cuando el alumno desee consultarlas. Por esta razón, las redes sociales se contemplan como potentes herramientas de comunicación en este campo.

La metodología propuesta se basa en la creación de comunidades de aprendizaje en entornos naturales, potenciando así el aprendizaje significativo de manera integrada de las áreas de conocimiento de Ciencias Sociales y Ciencias Naturales (frecuentemente estudiadas de manera inconexa), al igual que los investigadores autores del presente trabajo desarrollan su trayectoria científica en equipos interdisciplinares en el campo de los humedales. En las Facultades de Educación esta metodología implica un enriquecimiento de la concreción y desarrollo de los currículos educativos en las asignaturas de Didáctica del Conocimiento del Medio Natural, Social y Cultural, y en Educación Primaria en las áreas de conocimiento de las Ciencias Sociales y las Ciencias Naturales.

Las Tecnologías de la Información y la Comunicación (TIC) cobran especial relevancia en el proceso de enseñanza-aprendizaje, puesto que posibilitan la comunicación multidireccional, entre los alumnos, y no sólo unidireccional entre el docente y el discente, constituyendo una potente herramienta en los procesos formativos, de evaluación, de intercambio de información y metacognitivos. Además, la formación en estos aspectos contribuye al desarrollo de las competencias transversales asociadas, no limitándose únicamente a la consecución de competencias de las didácticas específicas.

Por otro lado, la transposición didáctica en las Facultades de Educación da lugar a potenciales líneas de investigación en este ámbito educativo por parte del mismo equipo docente que lleva a cabo las investigaciones objeto de la transposición. En el presente contexto socioeconómico, la importancia de unir campos de investigación optimizando los recursos disponibles es un aspecto muy interesante derivado de dicha metodología en estas facultades de marcada tradición docente y que ahora comienzan intensas trayectorias investigadoras.

La necesidad de trasladar las investigaciones a la docencia parte de la necesidad de revisar el currículo de acuerdo a la legislación educativa y de renovar el saber escolar, en concreto aquellos saberes denominados "envejecidos" y aquellos saberes que no se sabe muy bien por qué se enseñan de acuerdo a la sociedad actual (Chevallard, 
1985: 26). Estos saberes deben ser revisados y reemplazados por aquellos que constituyan respuestas a preguntas significativas para el alumno, que por otra parte no se limitan al ámbito de las didácticas específicas, sino que también incluyen aspectos relacionados con la educación ambiental, el conocimiento del mundo que rodea al alumno y la educación en valores. Estos aspectos no aparecen en muchos casos entre los saberes que se imparten en el aula, ni entre aquellos que le llegan al alumnado a través de los medios de comunicación que frecuentan. Pero cobran especial relevancia en una región como Castilla-La Mancha, de importante patrimonio medioambiental, y de climatología árida, donde las funciones y valores de los humedales son muy importantes, pero sin embargo poco conocidos y poco valorados.

Por tales motivos, se plantea en este trabajo una propuesta de transposición de la investigación sobre humedales al aula, en la que los medios de comunicación tienen un papel clave en el principal objetivo trazado: educar a personas competentes que contribuyan a la mejora del entorno en el que se desenvuelven.

\section{Metodología y desarrollo}

\subsection{Los humedales de la Reserva de la Biosfera de La Mancha Húmeda}

El punto de partida del presente trabajo son los humedales de la Reserva de la Biosfera de La Mancha Húmeda (RBMH), situada en el centro de Castilla-La Mancha (ver Figura 1) abarcando una extensión de unas 25.000 ha. La RBMH fue declarada en 1981 dentro del "Programa Hombre y Biosfera" de la UNESCO con la finalidad de preservar aquellas lagunas que la conforman respetando sus valores culturales y patrimoniales.

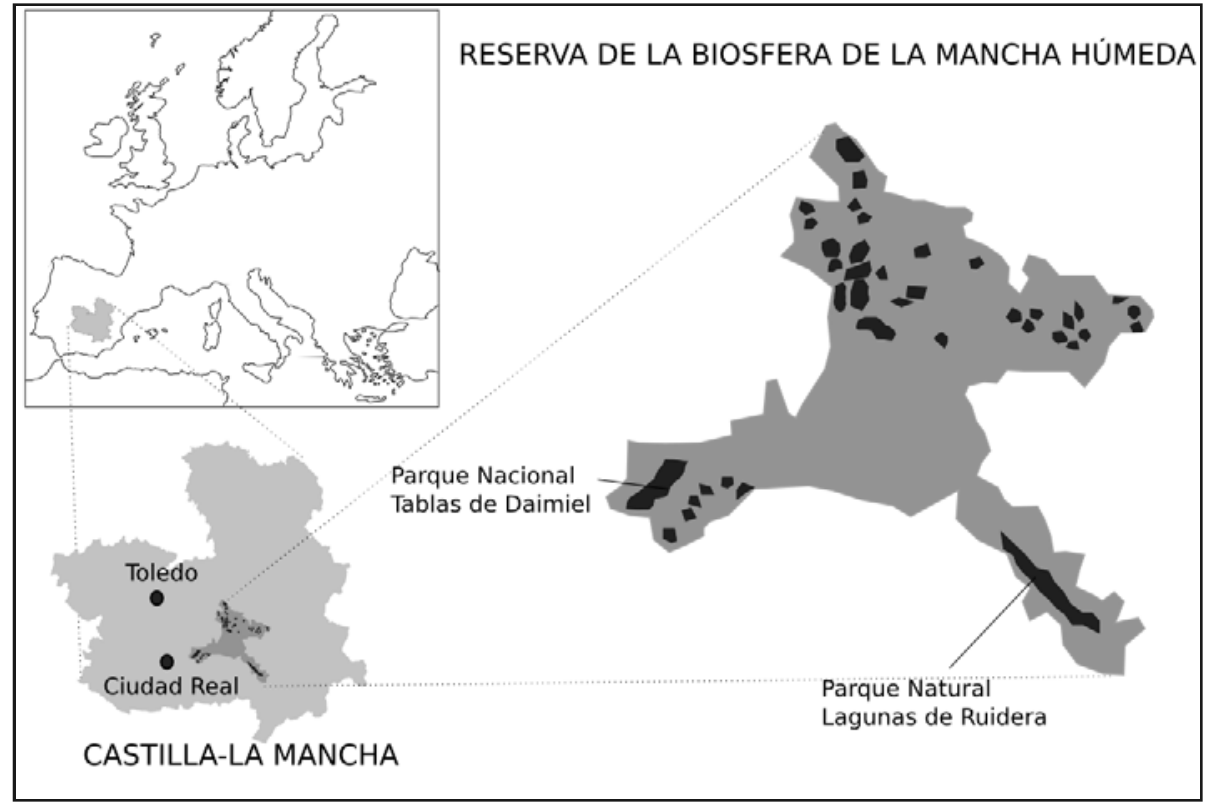

Figura 1. Situación de la Reserva de la Biosfera de La Mancha Húmeda. Fuente: adaptado de García Fernández et al. (2012) 
Castilla-La Mancha es una región que ha visto vulnerados gran parte de los ecosistemas protegidos que alberga durante las últimas décadas, fundamentalmente debido a acciones antropogénicas. Algunos ejemplos de estas acciones son la canalización de ríos, el vertido de efluentes de depuradora no tratados convenientemente y las extracciones intensivas de agua. Estas últimas han ocasionado importantes descensos en el nivel freático en el acuífero de Mancha Occidental, desconectando muchos de estos ecosistemas de sus aportes de agua subterránea (Custodio, 2002). Sin embargo, y pese a este deterioro existente, dichos ecosistemas responden positivamente a los procesos de restauración hidroecológica, como es el caso del Parque Nacional de Las Tablas de Daimiel (Navarro et al. 2011, 2012).

Los humedales son espacios de amplio valor educativo que engloban una gran diversidad de ecosistemas (Papapanagou et al., 2005), caracterizados por su gran variedad de funciones y valores (ambientales, sociales, económicos, etc.), lo que les convierte en áreas adecuadas para el planteamiento de actividades de enfoque holístico integrando las diferentes áreas de conocimiento (geomorfología, hidrología, biología, ordenación del territorio, etc.). La RBMH, recurso muy valioso y accesible, ofrece por todo lo anterior un amplio abanico de actividades significativas para el alumno (Pereira y Moreira Silva, 2010) que contribuye a que éste sea capaz de desenvolverse en el medio con responsabilidad, de manera que contribuya a su mejora y a su mantenimiento sostenible.

\subsection{Los humedales en la formación docente: transferencia de conocimiento}

La comprensión global del medio que rodea a los niños en sus primeras etapas educativas pasa por un estudio integrado de las materias de física, química, biología y geología, incluso de la música, las artes, la lengua y la literatura, y los humedales constituyen un elemento de unión de todas estas disciplinas.

Las investigaciones en el campo de estudio de los humedales pueden constituir el eje vertebrador de las materias del área de las Ciencias Experimentales y las Ciencias Sociales en las Facultades de Educación, aspecto muy interesante si consideramos que los alumnos que asimilan mejor las enseñanzas en ciencias son aquellos que perciben la ciencia como útil (Michaels et al., 2007). No hay que olvidar que precisamente las metodologías tradicionales de enseñanza de las ciencias basadas únicamente en transmisión de conocimientos teórico-prácticos y a menudo inconexos y sin aplicación práctica a la realidad suponen una falta de motivación en el alumnado (Ferreyra y González, 2000) que se traduce en malos resultados.

Estas investigaciones están relacionadas en muchos casos con problemáticas que son significativas para el alumnado, ya que en numerosas ocasiones los medios de comunicación se hacen eco de procesos de deterioro medioambiental como el del Parque Nacional de Las Tablas de Daimiel. La adquisición de una formación que les permita desarrollar una actitud crítica desde el conocimiento de la situación es un elemento clave en la formación de un futuro docente.

La metodología de trabajo es otro aspecto objeto de la transposición didáctica, y está basada en el método científico (usado en cualquier investigación). Transponer esta metodología de trabajo favorece la consecución de competencias como el pensamiento 
crítico y resolución de problemas, comunicación, colaboración y creatividad e innovación, que deben haber logrado los alumnos al final de su etapa universitaria (Partnership for $21^{\text {st }}$ Century Skills, 2010: 2). Mediante la transposición de esta metodología de trabajo también se trabajaría la consecución de los objetivos contemplados por el Committee on Conceptual Framework for the New K-12 Science Education Standards (Quinn et al., 2012), relacionados con la enseñanza de las ciencias: proponer cuestiones, desarrollar y utilizar modelos, planificar investigaciones, analizar e interpretar datos, utilizar TIC para la resolución de problemas, construir explicaciones, desarrollar argumentaciones basadas en evidencias y evaluar y comunicar los resultados.

En tercer lugar, además de los conocimientos y la metodología de trabajo, la conciencia medioambiental es otro aspecto que es objeto de transposición didáctica a los alumnos de las Facultades de Educación desde el estudio de evidencias científicas reelaboradas fruto de las investigaciones en humedales. Se trabaja a partir del estudio del deterioro y la conservación de los humedales, temática que en territorios como Castilla-La Mancha cobra especial relevancia, como ya se ha mencionado Los múltiples valores y funciones de estos ecosistemas, tanto ecológicos como sociales y culturales, son poco conocidos y, por tanto, poco valorados; por lo tanto, la transposición de la conciencia medioambiental tratada desde el prisma del conocimiento de los efectos de las acciones individuales en el entorno más próximo, a los que a veces se les resta importancia (Pellegrino y Hilton, 2012), es un punto clave dentro de este trabajo. La educación es el pilar fundamental sobre el que debe asentarse el tratamiento de la problemática medioambiental (Michaels et al., 2007) trabajando la modificación de los patrones individuales de comportamiento (Branagan, 2005), ya que es necesario el compromiso de toda la sociedad, además del de los gobiernos, para superar la crisis ambiental actual (Wilson, 1999).

Si atendemos a las legislaciones en materia educativa (Magntorn y Helldén, 2007) y en concreto a la de Castilla-La Mancha, encontramos los aspectos medioambientales destacados en el Decreto que regula la Enseñanza Primaria en esta comunidad autónoma (Decreto 68/2007) en los tres ciclos de la Educación Primaria. Para que los valores ambientales puedan transmitirse al aula de primaria, es necesario que se traten en las Facultades de Educación.

La propuesta de trabajo que aquí se plantea se concreta en una comunidad de aprendizaje basada en el diálogo (Jiménez et al., 2003), y en lo que Lemke (1997) denominó "hablar ciencias". Esta propuesta de transposición didáctica se basa en la atribución de significado a los conocimientos adquiridos, implicando la consecución de un "aprendizaje profundo": proceso por el cual el individuo es capaz de aplicar los conocimientos aprendidos a nuevas situaciones (transferirlos), y que a menudo requiere compartir conocimientos y la interacción entre los miembros implicados en el proceso de enseñanza-aprendizaje.

\subsection{Las redes de comunicación en la transferencia de conocimientos}

La comunicación, en sus distintos tipos, constituye uno de los elementos clave en cualquier transposición didáctica, puesto que es el mecanismo de transmisión de los conocimientos. En especial, los entornos multimedia y las redes de comunicación se 
han convertido en un conjunto de medios muy utilizados por los alumnos a través de los cuales éstos reciben información, resultándoles atractivos, y que constituyen nuevos contextos de aprendizaje dentro de la escuela, la casa o el lugar de trabajo del alumno. Ejemplos de estas herramientas son los blogs, los RSS y las plataformas de trabajo colaborativo, que permiten integrar la educación formal en un contexto que el alumno percibe como informal, de modo que se fijen mejor los conocimientos y se contribuya al aprendizaje profundo. Además, pueden ser utilizadas como foros de debate y diálogo y espacio donde compartir información dentro de la comunidad de aprendizaje.

La monitorización por parte del docente y del propio alumno del proceso de aprendizaje constituye un importante aspecto al que contribuyen los entornos multimedia y en concreto las herramientas de trabajo colaborativo (Newell, 1990). En referencia al proceso de monitorización por parte del docente, estas herramientas permiten que éste acceda al trabajo de sus alumnos evaluando sus errores en cuanto son detectados durante todo el proceso de enseñanza-aprendizaje, pudiendo realizar una evaluación formativa del mismo. Por otra parte, el alumno puede acceder mediante este tipo de herramientas al trabajo de sus compañeros, posibilitando una comunicación "peer to peer", que por otro lado se encuentra incluido dentro de la metodología de trabajo científico. De este modo, el alumno puede realizar críticas constructivas a estos trabajos, contribuyendo al desarrollo de la capacidad de crítica, autocrítica y autoevaluación (Duffy y Burns, 2006). El alumno se ve así inmerso en un proceso de reflexión metacognitiva paralelo a su proceso de enseñanza-aprendizaje. Estas herramientas multimedia permiten de este modo conseguir el aprendizaje profundo, tal y como han reflejado recientes investigaciones (Pellegrino y Hilton, 2012). Dicha comunicación multidireccional, en la que todos los participantes del proceso de enseñanza-aprendizaje reciben información del resto, sean profesores o alumnos, posibilita la creación de una comunidad de aprendizaje en la que se superan las barreras espacio-temporales y en la que el contexto de aprendizaje ya no se ciñe estrictamente al aula aprovechando el grado de autonomía que proporcionan estas herramientas (William, 2004).

La propuesta de transposición se concretaría mediante una adaptación de los resultados obtenidos en la investigación científica en humedales a los alumnos de las Facultades de Educación, extrayendo las relaciones de estos resultados con las competencias de grado. Además, se contempla la creación de una comunidad de aprendizaje en la que las redes sociales, y en concreto los grupos dentro de ellas, actúen como medio de intercambio de información.

En definitiva, el empleo de TIC como fuentes de información y medios de comunicación con objetivos pedagógicos claros, supone importantes mejoras en la calidad del proceso de enseñanza-aprendizaje en el ámbito de la reflexión metacognitiva, corrección de errores, aprendizaje profundo y transmisión de conocimientos en el proceso de transposición didáctica propuesto.

\section{Conclusiones}

En la sociedad actual, la optimización de recursos y de tiempo en la transmisión útil de conocimientos y de valores es fundamental, por lo que en el presente trabajo se re- 
aliza una reflexión acerca de cómo la transposición didáctica de la investigación en humedales es importante para la formación de los futuros maestros.

Tomar conciencia real del medio que les rodea y el desarrollo de valores, mediante el aprendizaje constante, son aspectos primordiales en las primeras etapas educativas. Para la consecución de estos objetivos, es fundamental el desarrollo de la capacidad de aprender a aprender, de reflexionar y de obtener información a partir de los medios de comunicación que tienen a su alcance. En concreto, los medios de comunicación que resultan motivadores al alumnado constituyen interesantes herramientas para obtener la información, contrastarla y compartirla, ya que de este modo el aprendizaje formará parte de su día a día al igual que lo son las redes sociales que utilizan para este y otros fines.

La transposición didáctica de los resultados de investigaciones en humedales aplicada a la docencia, implica dotar de significado a las enseñanzas que de manera tradicional se limitaban a transferir conocimientos a menudo obsoletos y a la realización de ejercicios de lápiz y papel, metodología que supone una de las causas del fracaso escolar en todas las etapas educativas, desde Primaria hasta las enseñanzas universitarias. Por ello es necesario plantear una reflexión acerca de la renovación de saberes obsoletos o que carecen de significación para el alumnado, siguiendo las indicaciones del Committee on Science Learning, Kindergarten through Eighth Grade (Duschl, 2007). El impacto que tiene la transferencia de la metodología de trabajo y la reelaboración de los contenidos fruto de las investigaciones en la docencia es muy positivo en la nueva redefinición del personal docente e investigador, desde la optimización del tiempo y recursos hasta la renovación de saberes obsoletos, dotación de significación a las distintas enseñanzas y el avance en el desarrollo de las competencias y del denominado "aprendizaje profundo" de las materias. Asimismo, esta línea de trabajo abre una vía paralela de investigación sobre la transposición didáctica en humedales en las Facultades de Educación, de marcada tradición docente pero relativamente reciente actividad investigadora.

Por otro lado, en un tema como son los ecosistemas protegidos, debemos destacar la importancia que tienen los valores y la conciencia medioambiental y que, al igual que los conocimientos y las metodologías de trabajo, también son susceptibles de ser adquiridos a partir de la transposición didáctica. En este proceso de transposición, no sólo el aula es el elemento clave dentro la adquisición de información. La metodología de trabajo, planteada dentro de una comunidad de aprendizaje, permite gracias a las TIC superar las barreras espacio-temporales mediante el uso de redes sociales, blogs, RSS, wikis y podcasts. De este modo el proceso de comunicación ya no es unidireccional entre docente y discente, sino que se posibilita una comunicación "peer to peer" entre todos y cada uno de los miembros de la comunidad de aprendizaje. Esta metodología ha demostrado ser muy efectiva en procesos de enseñanza-aprendizaje, ya que el discente comparte sus inquietudes e interrogantes para los que busca respuesta con su compañero, que las hace suyas, y comparte información y es evaluado, tomando conciencia de la evolución de su aprendizaje llevando a cabo un proceso de reflexión metacognitiva. 
Es destacable la importante repercusión que tiene el esfuerzo de la transposición didáctica de las investigaciones, no sólo en la eficiencia de los procesos de investigación-enseñanza-aprendizaje, mejorados al incluir aspectos como la transferencia de información reciente y novedosa mediante medios de comunicación motivadores, sino también al hacer que las investigaciones se optimicen debido a una doble y práctica finalidad docente (de profesores a futuros maestros). Se pretende contribuir a la mejora de la adquisición de competencias en la comunidad docente que contribuyan a mitigar la grave crisis ambiental, territorial y social actuales, en especial aquellas competencias que están directamente relacionadas con el medio ambiente y sus valores asociados: el pensamiento crítico, los medios de comunicación y las fuentes de información.

\section{Referencias bibliográficas}

BRANAGAN, Martin (2005): "Environmental education, activism and the arts." Convergence, 38 (4), pp. 43-50.

CHEVALLARD, Yves (1985): La transposition didactique; du savoir savant au savoir enseigné. París, La Pensée Sauvage.

Consejería de Educación y Ciencia, Junta de Comunidades de Castilla-La Mancha (2007): "Decreto 68/2007, de 29-05-2007, por el que se establece y ordena el currículo de la Educación Primaria en la Comunidad Autónoma de Castilla-La Mancha", en Diario Oficial de Castilla-La Mancha: http://edu.jccm.es/cp/n.s.rosario /decreto_primaria_clm.pdf [fecha de consulta: 24 de agosto de 2012]

CUSTODIO, Emilio (2002): “Aquifer overexploitation: what does it means?" $H y$ drogeology Journal 10 (2), pp. 254-277.

DUFFY, Peter D. y BRUNS, Axel (2006): "The Use of Blogs, Wikis and RSS in Education: A Conversation of Possibilities". Online Learning and Teaching Conference 2006, 26 Sep. 2006, Brisbane.

DUSCHL, Richard A.; SCHWEINGRUBER, Heidi A.; SHOUSE, Andrew W. (2007): Taking Science to School: Learning and Teaching Science in Grades K-8. Committee on Science Learning, Kindergarten through Eighth Grade; National Research Council. Washington D.C., The National Academies Press.

FERREYRA, Adriana y GONZÁLEZ, Eduardo M. (2000): "Reflexiones sobre la enseñanza de la física universitaria”. Enseñanza de las Ciencias, 18 (2), pp. 189-200.

GARCÍA FERNÁNDEZ, Beatriz; SÁNCHEZ VIZCAÍNO, Jesús y MATEOS JIMÉNEZ, Antonio (2012): "Los casos prácticos como actividades vertebradoras de las materias de Didáctica de las Ciencias Experimentales: el caso del Complejo Lagunar de Alcázar de San Juan (Ciudad Real)". Nuevos estándares en la innovación docente en Historia Natural. Actas del I Congreso de innovación docente universitaria en Historia Natural. Sevilla, Grupo de Investigación Didáctica de la Universidad de Sevilla, pp. 166-173. 
JIMÉNEZ ALEIXANDRE, María Pilar; CAAMAÑO, Aureli; OÑORBE, Ana; PEDRINACI, Emilio y DE PRO, Antonio (2003): Enseñar ciencias ( $1^{\mathrm{a}}$ ed.). Barcelona, Graó.

LEMKE, Jay L. (1997): Aprender a hablar Ciencia: Lenguaje, aprendizaje y valores. Barcelona, Paidós.

MAGNTORN, Ola y HELLDÉN, Gustav (2007): "Reading New Environments: Students' ability to generalize their understanding between different ecosystems". International Journal of Science Education, 29 (1), pp. 67-100.

MICHAELS, Sarah; SHOUSE, Andrew W. y SCHWEINGRUBER, Heidi A. (2007): Ready, Set, Science! Putting Research to Work in K-8 Science Classrooms. National Research Council. Center for Education, Division of Behavioral and Social Sciences and Education. Washington D.C., The National Academies Press.

NAVARRO, Vicente; GARCÍA, Beatriz; SÁNCHEZ, David y ASENSIO, Laura (2011): "An evaluation of the application of treated sewage effluents in Las Tablas de Daimiel National Park, Central Spain”. Journal of Hydrology 401 (2011), pp. 53-64.

NAVARRO, Vicente; GARCÍA, Beatriz y ASENSIO, Laura (2012): "Characterization of the infiltration rate in Las Tablas de Daimiel National Park, Central Spain". Hydrological Processes 26, pp. 367-378.

NEWELL, Allen (1990): Unified theories of cognition. Cambridge, Harvard University Press.

PAPAPANAGOU, Eirini; TINIAKOU, Argyro y GEORGIADIS, Theodoros (2005): "Environmental education in wetland ecosystems". Journal of Biological Education, 40 (1), pp. 25-30.

PELLEGRINO, James W. y HILTON, Margaret L. (editores) (2012): Education for Life and Work: Developing Transferable Knowledge and Skills in the $21^{\text {st }}$ Century. Committee on Defining Deeper Learning and $21^{\text {st }}$ Century Skills. Division of Behavioral and Social Sciences and Education; National Research Council. Washington D.C., The National Academies Press. Partnership for $21^{\text {st }}$ Century Skills. (2010): "2 $21^{\text {st }}$ Century Readiness for Every Student: A Policymaker's Guide": www.p21.org/documents/policymakersguide_final.pdf [fecha de consulta: 28 de junio de 2012]

PEREIRA FERREIRA, Critiane y MOREIRA SILVA, Meirelles Rosane (2010): "Participación de los alumnos en la construcción de un material didáctico: sus concepciones sobre el tema agua y salud como base para la preparación de las actividades". Enseñanza de las Ciencias, 28 (1).

QUINN, Helen; SCHWEINGRUBER, Heidi y KELLER, Thomas (editores) (2012): A Framework for K-12 Science Education: Practices, Crosscutting Concepts, and Core Ideas. Committee on Conceptual Framework for the New K-12 Science Education Standards; National Research Council. Washington D.C., The National Academies Press. 
VERRET, Michel (1975): Le Temps des études. París, Honorè Champion.

WILSON, Edward O. (1999): Consilience: The Unity of Knowledge. Londres, Abacus.

\section{Beatriz GARCÍA FERNÁNDEZ}

Profesora asociada, Departamento de Pedagogía. Facultad de Educación de Ciudad Real, Universidad de Castilla-La Mancha. Ronda de Calatrava nº 3, C.P. 13071, Ciudad Real. E-mail: Beatriz.Garcia@uclm.es.

\section{Gema SÁNCHEZ EMETERIO}

Profesora ayudante, Departamento de Geografía y Ordenación del Territorio. Facultad de Educación de Toledo, Universidad de Castilla-La Mancha. Avenida Carlos III s/n, Fábrica de Armas, C.P. 45071, Toledo. E-mail: Gema.Sanchez@uclm.es.

\section{David SÁNCHEZ RAMOS}

Profesor ayudante, Departamento de Ciencia y Tecnología Agroforestal y Genética. Escuela Técnica Superior de Ingenieros de Caminos, Canales y Puertos de Ciudad Real, Universidad de Castilla-La Mancha. Avenida Camilo José Cela, s/n, C.P. 13071, Ciudad Real. E-mail: David.SanchezRamos@uclm.es. 